\title{
Questions on Optimum Correction of Attenuation of Carrier Laser Beam Energy in Distributed Communication and Control Systems
}

\begin{abstract}
Questions on optimum correction of attenuation of intensity of carrier laser beam in distributed control and communication systems are considered. The model task on compensation of intensity of carrier laser beam in distributed control and communication systems is formulated. The task on optimum multiplicative correction of attenuation of intensity of carrier laser beam upon imposed limitation on power of used signals is formulated and solved. The applicability of obtained results for both the distributed laser communication system and laser targeting systems of controlled missiles is shown.

Index Terms - attenuation; compensation; optical
\end{abstract} communication, multiplicative correction.

\section{INTRODUCTION}

Different systems and complexes based on principle of propagation of carrier laser beam on far distances are encountered with attenuation of laser beam intensity on distance. Major reasons for attenuation of laser beam intensity in atmosphere are following[1]:

1.Absorption by atmospheric gases and attenuation due to effect of atmospheric aerosol.

2. Turbulence of atmosphere caused scattering of laser beam.

3. Effect of meteorological precipitation.

Most specific spheres of utilization of directed laser beam are following:

- Laser scanners and distance meters [2,3];

- Laser based optical communication [4,5]

- Transfer of energy on distance [6,7]

- Targeting of controlled missiles [8,9]

- Generation of optical noises for controlled missiles[10,11]

Such broad sphere of possible utilization of laser sources conditioned existence of different methods and techniques for accounting and compensation of laser beams intensity attenuation on distance. At the same time, these methods can be divided on following sub groups:

1.Methods of compensation of attenuation at transmitter.

2.Methods of compensation of attenuation at receiver.

In most cases of application of laser sources both methods can be used. At the same time the optimization procedures of these methods are quite different. The aim of this research is analysis conditions for optimization of methods used for compensation of laser beams intensity attenuation both at transmitter and receiver.

Abdulov R.N., candidate of technical sciences, Research Institute of Defence Industry Ministry of Azerbaijan Republic

\section{SUGGESTED METHOD}

Let us consider two models of distributed information network where channel forming laser beams are sent from transmitter of one cell to receiver of another one. (fig. $1 \mathrm{a}, \mathrm{b}$ ). First of all let us consider in brief the mechanism of attenuation of laser beam intensity in atmosphere. According to [12], power of laser beam when propagated through atmosphere decrease by exponential law in line with Bouguer-Beer law

$$
P(l)=P(0) \cdot e^{-\sigma l}
$$

(1)

where: $\mathrm{P}(l)$-luminous flux of laser radiation at distance 1 ; $\mathrm{P}(0)$ - luminous flux of laser radiation at source;

$\sigma-$ attenuation coefficient calculated on unit of distance as sum of absorption and scattering coefficients.

According [12], following wavelengths are used in technique of laser communication: $785 \mathrm{HM}, 850 \mathrm{HM}, 1550 \mathrm{HM}$. The reason is that factor of scattering should be dominant in total balance of attenuation of laser beam.

Following types of scattering should be differentiated:

1. Reley scattering, where $\mathrm{r}<<\lambda$; here $\mathrm{r}-$ radius of scattering particles in atmosphere.

In this case the scattering coefficient $\sigma_{\mathrm{p}}$ is defined as $\sigma_{\mathrm{p}}=\mathrm{A}_{1} \cdot \lambda^{-4} ; \mathrm{A}=\mathrm{const}$

$\lambda$ - wavelength of laser irradiation.

2. Mi scattering, where $r=\lambda$;

In this case

$$
\sigma=\mathrm{A}_{2} \cdot \lambda^{-1,6 \div 0} ; \mathrm{A}_{2}=\mathrm{const}
$$

3. Nom selective scattering, where $r>>\lambda$. In this case

$$
\sigma=A_{3} \cdot \lambda^{0}
$$

In this research we consider the case where $\lambda=$ const, i.e. $\sigma=$ const.

Let us consider the suggested method for optimization of above considered models of distributed control and communication systems using laser beam by correction of attenuation at receiver. Basic principles of suggested prosedure of optimization are follows:

1. We assume that following set exists

$$
\mathrm{L}=\left\{1_{\mathrm{i}}\right\} ; i=\overline{1, n}
$$

where: $\mathrm{l}_{\mathrm{i}}=\mathrm{l}_{\mathrm{i}-1}+\Delta \mathrm{l} ; \Delta \mathrm{l}=\mathrm{const} ; \quad i=\overline{1, n}$

2. In distributed system multiplicative correction of attenuation is carried out in line with following condition 
Questions on Optimum Correction of Attenuation of Carrier Laser Beam Energy in Distributed Communication and Control Systems

$$
\sum_{i=1}^{n} x_{i} \cdot y\left(x_{i}\right)=C \quad \Phi_{1}=\int_{0}^{x_{\max }} x \cdot y(x) d x=C
$$

where: $\mathrm{x}_{\mathrm{i}}-$ attenuated signals arriving at the input of

The geometrical meaning of condition (2) is shown in fig. 3 . receiver passing distance $l_{\mathrm{i}}$ between transmitter and receiver;

$\mathrm{y}\left(\mathrm{x}_{\mathrm{i}}\right)$ - signal of multiplicative correction in form of function of argument $\mathrm{x}_{\mathrm{i}}$.

Formula (1) can be written in continuous form as

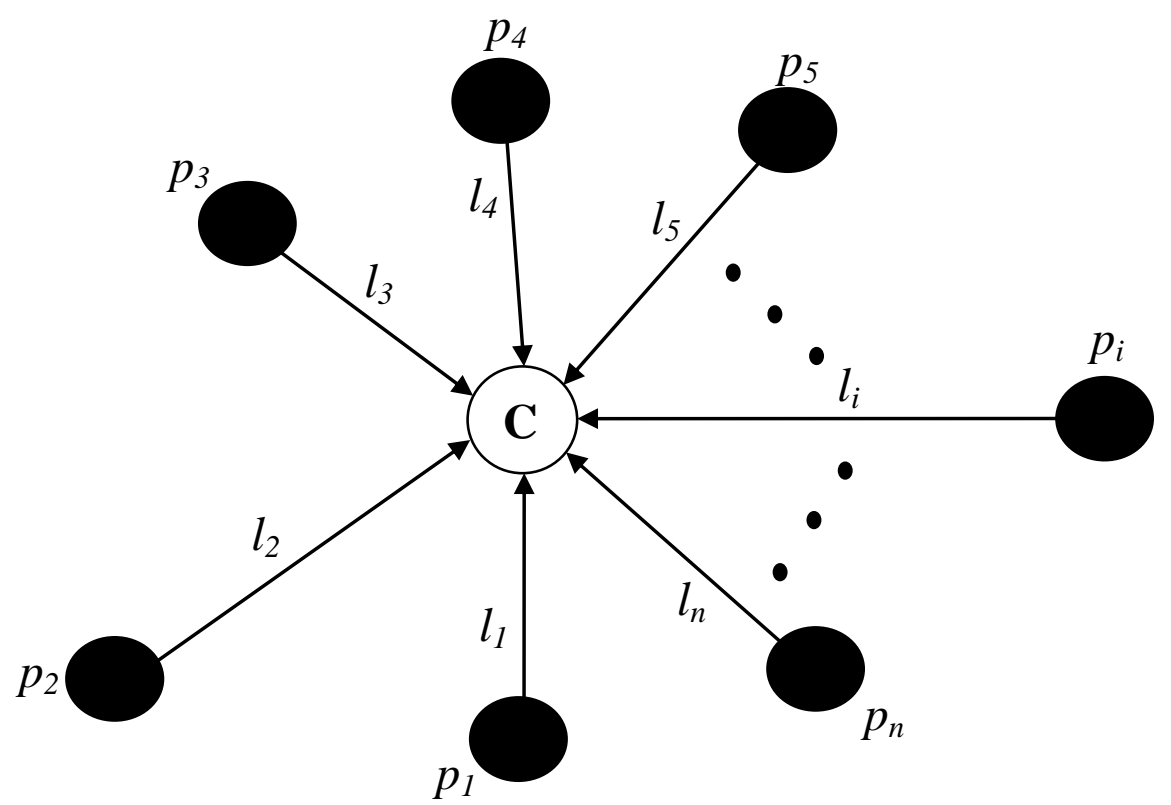

Fig1. Researched model of receipt of optical signals at the cell $\mathrm{C}$ from set of transmitting cells $\left\{\mathrm{P}_{\mathrm{i}}\right\} ; i=\overline{1, n}$

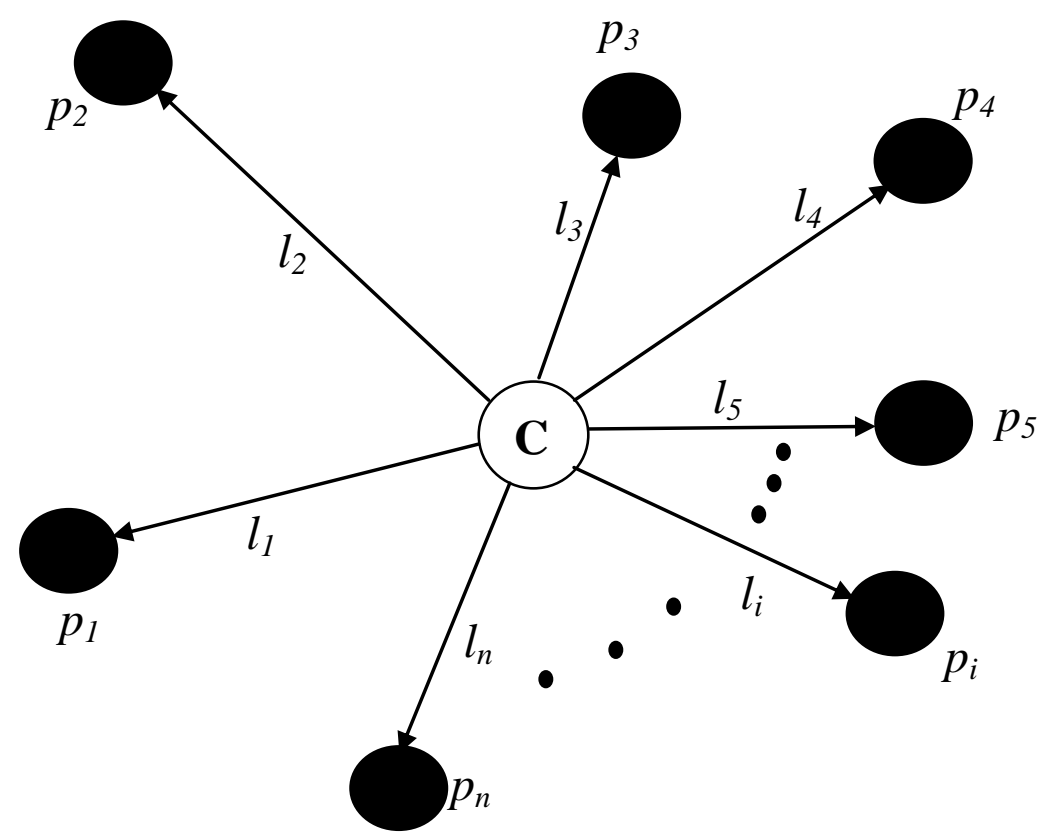

Fig. 2. Researched model of receipt of optical signals sent from cell $\mathrm{C}$ to set of receiving cells $\left\{\mathrm{P}_{\mathrm{i}}\right\} ; i=\overline{1, n}$ 


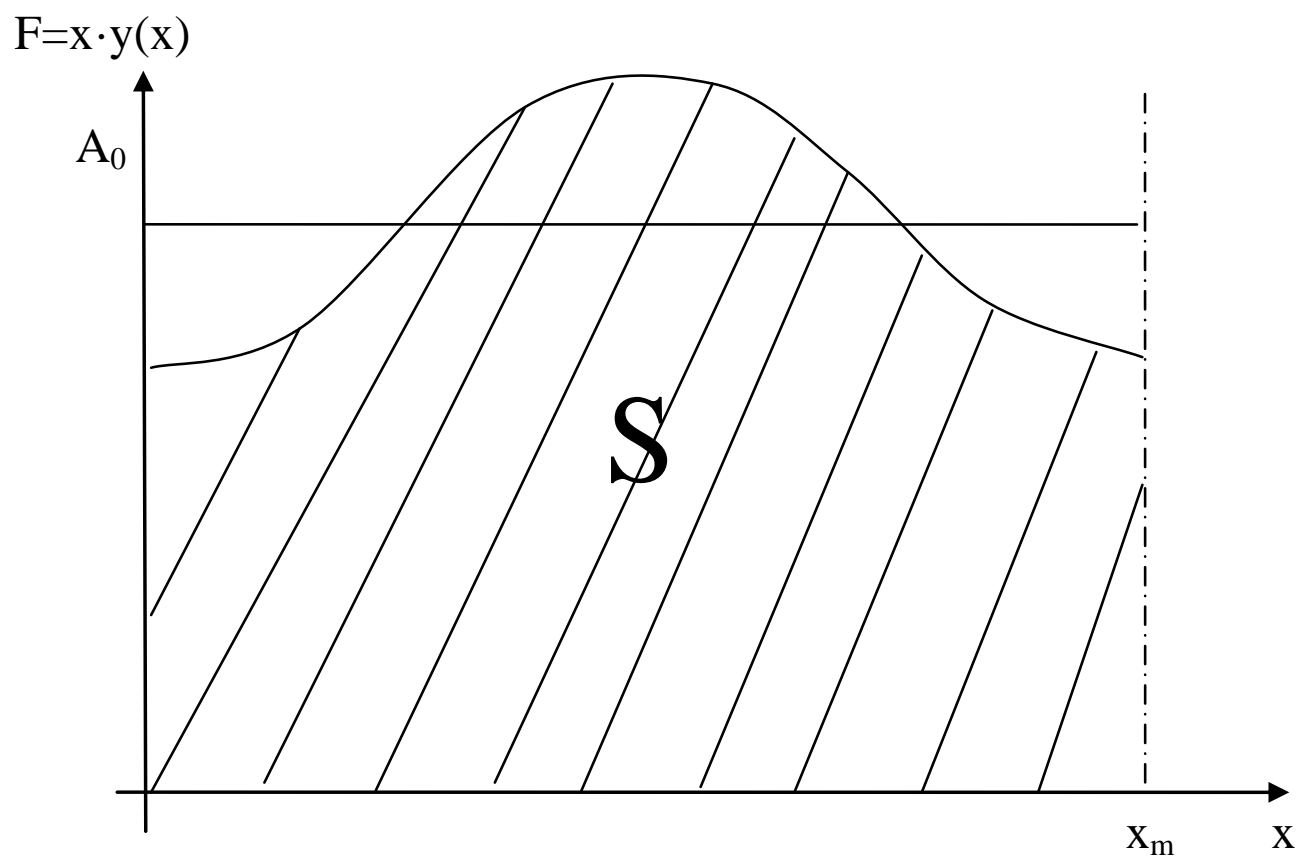

Fig. 3. Geometrical meaning of condition (2), that is equality $A_{0} \cdot x_{m}=S=C$, where $S$ is square of area situated under curve of function $\mathrm{F}=\mathrm{x} \cdot \mathrm{y}(\mathrm{x})$

3. Following energetic requirements are imposed on signals $\mathrm{F}_{1}=\mathrm{x}$ and $\mathrm{F}_{2}=\mathrm{y}(\mathrm{x})$

$$
\Phi_{2}=\int_{0}^{x_{\max }}\left[x^{2} \cdot y(x)^{2}\right] d x \rightarrow \min
$$

(3)

Physical meaning of condition (3) is that energy consumption in distributed system should be minimum.

4. Following task is formulated: To formulate optimum correcting function $\mathrm{y}(\mathrm{x})$ upon which following functional of non- conditional variation optimization could reach its minimum value

$$
F_{0}=\lambda \cdot \Phi_{1}+\Phi_{2}
$$

где: $\lambda$ - Lagrange multiplier.

Taking into account formulas (2), (3), (4) we have

$$
F_{0}=\lambda \cdot \int_{0}^{x_{\max }} x \cdot y(x) d x+\int_{0}^{x_{\max }}\left[x^{2} \cdot y(x)^{2}\right] d x
$$

In order to calculate the optimum function $\mathrm{y}(\mathrm{x})$, leading $\mathrm{F}_{0}$ to its extremum we use Euyler-Lagrange equation, in line which searched for function $\mathrm{y}(\mathrm{x})$ should meet following condition

$$
\frac{d\left\{\lambda \cdot x \cdot y(x)+x^{2} \cdot y(x)^{2}\right\}}{d y(x)}=0
$$

From condition (6) we get

$$
\lambda \cdot x+2 y(x)=0
$$

From formula (7) it could be found that

$$
y(x)=-\frac{\lambda \cdot x}{2}
$$

Taking into account (2) and (8) we have

$$
-\int_{0}^{x_{\max }} x \cdot \frac{\lambda \cdot x}{2} d x=C
$$

From equation(9) we can find that

$$
\lambda=-\frac{6 C}{x_{m}^{3}}
$$

$$
x_{m}
$$

Taking into account formulas (7) and (10) it could be found that

$$
\frac{3 C \cdot x}{x_{m}^{3}}=y(x)
$$

Thus upon solution (11) the target functional reaches extremum. In order to determine the type of extremum the second derivative functional (5) integrant on $\mathrm{y}(\mathrm{x})$ should be calculated. As a result we can find that

$$
\frac{d^{2}\left\{\lambda \cdot x \cdot y(x)+x^{2} \cdot y(x)^{2}\right\}}{d y(x)^{2}}=2
$$

Consequently, upon solution (11) target functional (5) reaches its minimal value.

\section{DISCUSSION}

Thus in line with solution (11) in distributed optical laser sets of control and communications compensation of attenuation 
of signal power should be carried out using method of adaptive multiplicative correction where correction signal is directly proportional power of received signal

Above optimization model is applicable also for correction realized at transmitter.

In this case all formulas $(1) \div(12)$ are valid ones but $\mathrm{x}$ would mean not received signal at the input of receiver, but output signal of transmitter.

As for applicability of results of held model research for laser targeting systems of guided missiles, these results are applicable in case of consideration of targets situated at different distances from laser beam source by correction at the output of laser emitter.

\section{CONCLUSION}

In conclusion following major results of held research can be formulated:

1. The model task on compensation of attenuation of laser beam energy in distributed systems of control and communication is formulated.

2. The task on optimum multiplicative correction of laser beams energy attenuation upon imposing the limitation condition on power parameters of used signals is formulated and solved.

3. The applicability of obtained results in distributed laser communication system also in laser targeting systems of guided missiles is shown.

\section{REFERENCES}

[1] Abdulsalam Alkholidi and Khalil Altowij. Effect of Clear Atmospheric Turbulence on Quality of Free Space Optical Communications in Western Asia https://www.intechopen.com/

[2] Szczepanski J., Orczykowski T., Tocarcryk T. Using 3D terrestrial Laser scanning for surveying hydrotechnical structures when preparing flood Hazard Maps. Pol J. Environ. Stud. Vol. 21, no. 5, $2012,1405-1412$.

[3] Jackowski W. Applications of laser distancemeters disto to control surveys of mine headings. Geodezja tom 12 zeszyt 1, 2006.

[4] Amit Agrawal, Gaurav Kumar, Maheshwari Narayan Singh, Pradeep Kumar, Pransu Mathur. Data Transmission Using Laser Light. International Journal of Advanced Computer Technology (IJACT).

[5] M. Taslakov, V. Simeonov, H. van den Bergh, "Line-of-sight data transmission system based on mid IR quantum cascade laser", Proc. SPIE 6877, Free-Space Laser Communication Technologies XX, 68770F (13 February 2008); doi 10.1117/12.763731; https://doi.org/10.1117/12.763731

[6] Carlo De Santi, Matteo Meneghini, Alessandro Caria, Ezgi Dogmus , Malek Zegaoui, Farid Medjdoub, Boris Kalinic, Tiziana Cesca, Gaudenzio Meneghesso and Enrico Zanoni. GaN-Based Laser Wireless Power Transfer System. Materials 2018, 11, 153; doi:10.3390/ma11010153 www.mdpi.com/journal/materials

[7] Rajnish Saxena, Poonam Bakolia, Dalipkumar. Wireless Power Transmission - The Future of Power Transmission System. Journal of Advanced Computing and Communication Technologies (ISSN: 2347 - 2804) Volume No.5 Issue No.3, June 201748

[8] IOSR Journal of Engineering (IOSRJEN) www.iosrjen.org ISSN (e): 2250-3021, ISSN (p): 2278-8719 Vol. 05, Issue 01 (January. 2015), ||V3|| PP 15-25 International organization of Scientific Research 15 |P a g e Laser Based Guidance System For Projectile Gayathri. N

[9] M. R. Akhila S. Gopika R. J. Abraham Application of a Complex Lead Compensator for a Laser Guided Missile. Journal of The Institution of Engineers (India): Series C January 2013, Volume 94, Issue 1, pp 37-41 https://doi.org/10.1007/s40032-013-0058-0

[10] H.H.P.Th. Bekman, J.C. van den Heuvel, F.J.M van Putten, H.M.A. Schleijpen. Development of a Mid-Infrared Laser for Study of Infrared Countermeasures Techniques Technologies for Optical Countermeasures, edited by David H. Titterton, Proceedings of SPIE Vol. 5615 (SPIE, Bellingham, WA, 2004) 0277-786X/04/\$15 · doi: $10.1117 / 12.578214$
[11] Ahmed Sami Bahgat, Ashraf F. El-Sherif, Yaser H. El-Sharkawy, "Design and implementation of a deception jamming system for laser receivers", Proc. SPIE 9768, Light-Emitting Diodes: Materials, Devices, and Applications for Solid State Lighting XX, 97681M (8 March 2016); doi: 10.1117/12.2206011; https://doi.org/10.1117/12.2206011

[12] Isaac I. Kim, Bruce McArthur, Eric J. Korevaar, "Comparison of laser beam propagation at $785 \mathrm{~nm}$ and $1550 \mathrm{~nm}$ in fog and haze for optical wireless communications", Proc. SPIE 4214, Optical Wireless Communications III, (6 February 2001); doi: 10.1117/12.417512; https://doi.org/10.1117/12.417512 